\title{
Stroke in systemic lupus erythematosus: a Swedish population-based cohort study
}

Elizabeth V. Arkema, Elisabet Svenungsson, Mia Von Euler, Christopher Sjöwall and J ulia F. Simard

The self-archived postprint version of this journal article is available at Linköping University Institutional Repository (DiVA):

http:/ / urn.kb.se/ resolve?urn=urn:nbn:se:liu:diva- 140790

N.B.: When citing this work, cite the original publication.

Arkema, E. V., Svenungsson, E., Von Euler, M., Sjöwall, C., Simard, J . F., (2017), Stroke in systemic lupus erythematosus: a Swedish population-based cohort study, Annals of the Rheumatic Diseases, 76(9), 1544-1549. https:// doi.org/ 10.1136/ annrheumdis-2016-210973

Original publication available at:

https:// doi.org/ 10.1136/ annrheumdis-2016-210973

Copyright: BMJ Publishing Group

http:// group.bmj.com/ 


\section{Stroke in Systemic Lupus Erythematosus: A Swedish population-based cohort study}

Authors: Elizabeth V. Arkema ${ }^{1}$, Elisabet Svenungsson ${ }^{2}$, Mia von Euler ${ }^{3}$, Christopher Sjöwall $^{4}$, Julia F. Simard ${ }^{1,5,6}$

\section{Affiliations}

${ }^{1}$ Clinical Epidemiology Unit, Department of Medicine Solna, Karolinska Institute, Stockholm, Sweden

${ }^{2}$ Rheumatology Unit, Department of Medicine, Karolinska University Hospital, Karolinska Institutet, Stockholm, Sweden

${ }^{3}$ Karolinska Institutet Stroke Research Network at Södersjukhuset, Department of Clinical Science and Education, Karolinska Institutet, Stockholm, Sweden

${ }^{4}$ Rheumatology/Division of Neuro and Inflammation Sciences, Department of Clinical and Experimental, Medicine, Linköping University, Linköping, Sweden

${ }^{5}$ Division of Epidemiology, Department of Health Research \& Policy, Stanford School of Medicine

${ }^{6}$ Division of Immunology \& Rheumatology, Department of Medicine, Stanford School of Medicine

\section{Corresponding Author}

Elizabeth Arkema, ScD

Department of Medicine Solna, Clinical Epidemiology Unit T2

Karolinska Institutet

17176 Stockholm Sweden

Elizabeth.Arkema@ki.se

Word count [manuscript max 3000]: 3050

References [max 50]: 25

Keywords: systemic lupus erythematosus, epidemiology, cardiovascular disease

Running header Stroke in Systemic Lupus Erythematosus 


\section{ABSTRACT}

Objective To study the occurrence of ischaemic and haemorrhagic stroke in systemic lupus erythematosus (SLE) compared to the general population by age, sex, and time since SLE diagnosis. Methods Adults with incident SLE were identified from the Swedish National Patient Register (NPR, $\mathrm{N}=3390$ ) and general population comparators from the Total Population Register were matched on age, sex and county ( $\mathrm{N}=16730$ ). Individuals were followed prospectively until first of death, Dec 2013, emigration or incident stroke (identified from the NPR, Cause of Death Register and the Stroke Register). Incidence rates, rate differences and hazard ratios (HR) were estimated comparing SLE to non-SLE. Estimates were stratified by sex, age and time since diagnosis.

Results We observed 126 strokes in SLE and 304 in the general population. Individuals with SLE had a two-fold increased rate of ischaemic stroke compared to the general population ( $\mathrm{HR} 2.2 ; 95 \% \mathrm{Cl} 1.7$, 2.8). The HR for intracerebral haemorrhage was $1.4(95 \% \mathrm{Cl} 0.7,2.8)$. There was effect modification by sex and age, with the highest HRs for females and individuals less than 50 years old. The HR for ischaemic stroke was highest in the first year of follow-up $(3.7 ; 95 \% \mathrm{Cl} 2.1,6.5)$.

Conclusions The relative risk of ischaemic stroke in SLE was more than doubled compared to the general population, and importantly, the highest relative risks were observed within the first year after SLE diagnosis. Thus the first encounter with patients presents an opportunity for rheumatologists to screen for risk factors and intervene.

Abstract word count [max 250]: 243 


\section{INTRODUCTION}

Systemic lupus erythematosus (SLE) is a complex autoimmune disease with heterogeneous presentation which is associated with an increased risk of cardiovascular disease.[1, 2] Stroke has been investigated in some previous studies as a composite endpoint with cardiovascular disease, but given that it is an important cause of morbidity and mortality in SLE and has a different etiology, a separate investigation is warranted.[3] Traditional risk factors, such as hypertension which is more common in SLE, likely contribute to the increased risk of cerebrovascular disease. SLE-specific factors including pro-inflammatory cytokines, pro-thrombotic antiphospholipid antibodies, impaired renal function and exposure to medications like glucocorticoids may also play important roles.[4-7] A recent meta-analysis reported a two-fold increased risk of ischaemic stroke in SLE compared with the general population.[8] The relative risk of intracerebral haemorrhage was estimated to be almost three-fold, but this was based on only three studies.[9-11] Few studies have examined the relative risk of stroke stratified by age, sex or disease duration.[9-11] Absolute rates are seldom reported, which are especially necessary for communicating risk to young individuals for whom the outcome is rare.

In the general population, treatment of hypertension and lifestyle modifications have been shown to substantially reduce the risk of stroke.[12,13] Individuals with SLE who have a higher risk of stroke may greatly benefit from early intervention. Understanding who is at the highest risk of stroke and when the highest risk occurs could not only help to target groups of people for preventative treatment but also provide clues to the underlying drivers of stroke in SLE. Our aim was to study the occurrence of ischaemic and haemorrhagic stroke in SLE compared to the general population by age, sex, and time since diagnosis.

\section{METHODS}

We used Swedish national registers and a matched cohort study design to compare the risk of incident stroke in individuals with SLE to those without SLE.

\section{Study population}

A population-based SLE cohort was identified using the National Patient Register (NPR), which contains data on inpatient care 1964-2013 (nationwide since 1987) and outpatient, non-primary care 2001-2013. We included individuals 18 years or older with at least two discharge diagnoses listing an ICD code for SLE, one or more registered with a relevant specialist (rheumatology, dermatology, nephology, internal medicine, or pediatrics). This definition has been shown to result in an accurate classification of SLE in these data.[14] To best identify newly diagnosed (incident) SLE, we required 
that individuals have no inpatient or outpatient SLE-coded visits for at least two years before they were first identified with an SLE ICD code. Individuals were included if they received their first ever SLE ICD code Jan 2003 or later thus allowing for at least two years of outpatient data.

For each individual with SLE, five comparators were selected from the Total Population Register matched on sex, year of birth and residential county. These general population comparators (nonSLE) were required to be living in Sweden at the time their matched SLE case fulfilled the case definition (index date) and were eligible to become cases later during follow-up.

\section{Covariates}

Information from several national registers was retrieved and linked to the study population using each individual's unique personal identification number. Education was obtained from the Education Register and categorized into $\leq 9$ years, $10-12$ years, $>12$ years of education and missing. Country of birth was obtained from the Total Population Register (categorized as Nordic, non-Nordic Europe and outside Europe). Information on comorbidities was collected from the NPR. Indicator variables were created if any visit occurred before start of follow-up listing an ICD code for hypertension, congestive heart disease, diabetes, atrial fibrillation and antiphospholipid syndrome (APS), see Supplementary Table 1 for details.

\section{Stroke Identification}

Date and type of stroke (ischaemic stroke, intracerebral haemorrhage, subarachnoid haemorrhage, unspecified) were identified using ICD codes from three sources (see Supplementary Table 1 for ICD codes used):

1) The NPR; both hospitalizations and outpatient non-primary visits listing an ICD code for stroke either as main diagnosis or contributory diagnosis. ICD codes for stroke from the NPR have been shown to be valid (PPV 94.0\%).[15]

2) The Swedish Stroke Register (Riksstroke); a National quality register that covers all hospitals admitting acute stroke patients with excellent data on stroke subtype (PPV $\geq 95 \%$ ).[16] Riksstroke does not contain information on subarachnoid haemorrhage.

3) The Cause of Death Register; date of death and main and contributory causes for almost $100 \%$ of all deaths in Sweden was used to identify fatal strokes (PPV 87.3\%).[15]

Some strokes were listed in both the NPR and in Riksstroke $(n=259)$. If the diagnoses from the two sources were different, the Riksstroke diagnosis was considered the gold standard and the stroke was reclassified accordingly (seven unspecified strokes were reclassified as ischaemic and one ischaemic stroke was reclassified as intracerebral haemorrhage). 


\section{Follow-up}

Start of follow-up began on the date of the second SLE-coded visit or index date and ended at incident stroke, emigration date (retrieved from the Total Population Register), death or 31 December 2013, whichever came first. Individuals with a history of stroke at start of follow-up were excluded from all analyses. 3,562 individuals with SLE and 17,062 general population comparators were identified before excluding those with a history of stroke. $172(4.8 \%)$ individuals with SLE had a history of stroke at start of follow-up compared to $332(1.9 \%)$ in the general population.

\section{Statistical Analysis}

Incidence rates (IRs) of stroke per 1,000 person-years (py) were calculated. Age- and sexstandardized rate differences with 95\% confidence intervals comparing SLE and non-SLE were estimated. Age- and sex-adjusted Cox models were used to estimate the hazard ratio and 95\% confidence interval $(\mathrm{HR} 95 \% \mathrm{Cl})$ for stroke comparing SLE to non-SLE. Models were additionally adjusted for history of hypertension, congestive heart disease, atrial fibrillation and educational level. The proportional hazards assumption was tested by calculating the $p$-value for the interaction term between log of follow-up time and SLE. A p-value $<0.05$ was considered statistically significant. Models were stratified by sex, age at start of follow-up $(<50,50-59, \geq 60 \mathrm{y})$, and time since start of follow-up (a proxy for SLE disease duration; $<1,1-<5,5-11$ y). Stratification categories were collapsed if fewer than five events occurred in a stratum and if there still remained strata with fewer than five individuals, numbers were not reported and no IRs or HRs were calculated. Effect modification by sex, age and time since start of follow-up was tested using the likelihood ratio test (LRT).

\section{Sensitivity analyses}

Although using two ICD codes for SLE including at least one from a specialist clinic from the NPR has been shown to identify individuals with SLE accurately, some misclassification may still remain.[14] Therefore, we restricted to a subset of the SLE population who were enrolled in one of two clinical SLE cohorts in Sweden (Linköping and Stockholm) and had 4 or more ACR criteria for SLE fulfilled. In this analysis, start of follow-up began on the date of inclusion in their respective clinical cohort or January 1,2003 , whichever came later, to make the study period comparable to the main analysis. Follow-up ended at incident stroke, emigration, death or end of follow-up (31 Dec 2011 for the Linköping Cohort and 31 Dec 2013 for the Stockholm Cohort), whichever came first. Unlike the primary nationwide analysis, these data include individuals with prevalent SLE. More information on these cohorts can be found elsewhere.[17, 18] 
Because we estimate cause-specific HRs in our primary analyses, which assume that the relative hazards of stroke and death are independent, we also calculated HRs using Fine and Grey competing risk regression models to account for the competing risks of other stroke types and mortality.[19] Lastly, HRs were recalculated restricting the study population to individuals without a history of APS at start of follow-up and censored at APS diagnosis during follow up to determine whether our results were driven by APS. Because this ICD code has not been validated, we also re-ran analyses restricted to data from the Stockholm clinical cohort excluding those with APS recorded in medical records.

\section{RESULTS}

The study population included 3,390 individuals with SLE and 16,730 general population comparators. Eighty-five percent of the study population was female and the mean age at start of follow-up was 49 years (Table 1). A higher proportion of individuals with SLE had a history of hypertension, atrial fibrillation, diabetes and congestive heart disease compared to the general population (Table 1). During the study period, 126 incident strokes occurred in SLE, 304 strokes occurred in non-SLE and approximately three-fourths of strokes were ischaemic in both SLE and nonSLE. When excluding subarachnoid haemorrhage and unspecified stroke, $87 \%$ of strokes were ischemic, which is similar to the national average.[20] Mean age at stroke was younger in SLE compared to non-SLE (68.4 vs. 73.3; Table 2). Fourteen percent of strokes in SLE occurred before the age of 50 compared to $4 \%$ in the general population. A higher proportion of individuals with stroke were female in SLE compared to the general population (79\% vs. $68 \%$ ).

\section{Association between SLE and stroke}

The incidence rate of any stroke was 7.7 per 1,000 py $(95 \% \mathrm{Cl} 6.5,9.2)$ in SLE and 3.5 per 1,000 py $(95 \% \mathrm{Cl} 3.2,4.0)$ in their age- and sex-matched general population comparators. The age- and sexadjusted rate difference was 4.4 strokes per 1,000 py $(95 \% \mathrm{Cl} 3.0,5.9)$. Individuals with SLE had a two-fold increased hazard of ischaemic stroke compared to the general population (HR $2.2 ; 95 \% \mathrm{Cl}$ 1.8, 2.8; Table 3). The test of non-proportionality was statistically significant for analyses of ischaemic stroke, therefore the HR stratified by follow-up time may better represent the association between SLE and ischaemic stroke. The HRs for intracerebral and subarachnoid haemorrhage were HR 1.4 $(95 \% \mathrm{Cl} 0.7,2.9)$ and HR $1.4(95 \% \mathrm{Cl} 0.5,3.9)$, respectively. The risk of unspecified stroke was higher in the SLE population than in the general population (HR $2.795 \% \mathrm{Cl} 1.3,5.6$ ).

Association between SLE and stroke according to age, sex and time since diagnosis 
Stratified analyses were only conducted for the ischaemic stroke and intracerebral haemorrhage outcomes due to limited power for the other types of stroke. There was statistically significant effect modification by sex and age, with the highest HRs for females and individuals less than 50 years old. Although the relative risk was highest for the youngest age groups, the absolute rate was highest among the oldest (for those aged 60 or older, 9 additional ischaemic strokes per 1,000 years occurred in the SLE group compared to the non-SLE group). The HR for ischaemic stroke was highest in the first year of follow-up and effect modification by time was observed (Table 4).

For intracerebral haemorrhage, there were fewer than five events in males with SLE, therefore estimates were not calculated for this stratum. Age and follow-up time categories were collapsed due to small numbers. The highest HR was in individuals $<60$ years old, based on only 5 events in the SLE group and 8 in the non-SLE group. There was no effect modification by time since start of followup but there was significant modification by age with the highest HR occurring in the youngest age group.

\section{Sensitivity analyses}

Analyses restricted to clinically confirmed SLE from the Linköping and Stockholm cohorts resulted in similar HR estimates for all stroke and ischaemic stroke (Supplementary Table 2). Numbers were too small to estimate HRs for other types of stroke in this subset. After excluding individuals with an APS diagnosis code at start of follow-up and censoring on date of APS diagnosis code if it was received during follow-up, the results were similar. Using the higher quality APS diagnosis from the Stockholm cohort to exclude individuals with APS in this subset, the results were also similar to the main analyses (Supplementary Table 2). HRs from competing risks models were similar to those from cause-specific models, although slightly lower (Supplementary Table 3).

\section{DISCUSSION}

Individuals with SLE have twice the risk of ischaemic stroke compared to the general population and the relative risk differed by age and sex, with a higher relative risk in females and individuals younger than 50 years of age. The relative risk of ischaemic stroke in SLE was increased within the first year after diagnosis and remained relatively constant over up to 11 years of follow-up. This demonstrates that early in the disease course, SLE patients are already at increased risk of stroke and the first encounter with patients represents an opportunity for rheumatologists to intervene. The relative risk of intracerebral haemorrhage was also higher at a younger age in the SLE group compared to nonSLE. The more time since SLE diagnosis, the higher the relative risk of intracerebral haemorrhage. This may be due to a cumulative effect of inflammation or medications used more often in SLE such as anticoagulants. 
Although young individuals with SLE were at a higher relative risk of stroke, stroke before the age of 60 was not common and the rate of stroke in SLE was low. In fact, the highest rate of stroke was observed in older SLE patients ( $\geq 60$ years old at incident SLE; 17 ischaemic strokes per 1,000), indicating that prevention of stroke in this vulnerable group would make an important impact on this patient population.

There are several factors which likely contribute to the increased stroke risk associated with SLE. There exists a large inflammatory burden in SLE which causes endothelial activation leading to the development of atherosclerosis and thrombus formation.[7] In this setting, an immunological challenge such as an infection or a lupus flare, may result in stroke.[21] Risk factors that predict stroke in the general population do not appear to accurately predict stroke in SLE. $[22,23]$ This suggests that an SLE-specific risk score, with evidence-based guidelines for stroke prevention, is necessary to decrease the excess risk we observe in these patients. This should be studied in a large cohort of individuals with SLE with prospective follow-up and time-varying measurements of risk factors easily obtained and/or available in most clinics.

Our findings are similar to previous studies of overall stroke and ischaemic stroke, which report between a 2- to 3-fold increased risk associated with SLE. However, our estimated relative risk is lower for intracerebral haemorrhage and subarachnoid haemorrhage compared to some previous studies.[9-11] One explanation for this difference is that we identified individuals with SLE from both inpatient and outpatient care, whereas previous studies on haemorrhagic stroke have identified SLE solely from hospitalization data. Previous studies may have included SLE cases with a greater disease severity and/or more comorbidities compared to those seen in outpatient care. If we were to restrict our SLE population to those with at least one hospitalization for SLE, the HR for haemorrhagic stroke would increase from 1.4 to 2.5 and reach a similar magnitude as previous reports.

Some limitations of our study should be mentioned. The date of second SLE-coded visit was used as date of incident disease, which is likely misclassified if it takes some time to be diagnosed with SLE. When comparing date of diagnosis in the cases included in the Stockholm and Linköping cohorts with first date registered in the NPR, over $90 \%$ of individuals were registered within two years of first clinical cohort diagnosis. In our study, the median time between first and second SLE diagnosis (when criteria for inclusion were fulfilled) was three months. We do not have data on first encounter in primary care. If a stroke occurred soon after first SLE diagnosis before a second SLE diagnosis in nonprimary care was given, these individuals were excluded. This minimizes SLE misclassification by requiring specialist visits with SLE, but we may have missed early strokes, which would lead to an underestimation of the risk in the first year after diagnosis. 
Some strokes could have been misclassified, especially in the SLE group if a neurological manifestation is diagnosed at first as a stroke. The Patient Register and the Cause of Death Register may have some misclassification of stroke subtype, and the accuracy of subarachnoid haemorrhage codes have not been investigated. We minimized misclassification of stroke through the use of the Riksstroke register, which has high quality data on acute stroke.[15]

We could not take into account smoking or obesity in this study, though it is unlikely that these would entirely explain our findings. How medications and disease activity play a role in stroke occurrence was not addressed, but examining these factors as chronic exposures or triggers is an important next step which requires a different study design. Lastly, we attempted to exclude individuals with a diagnosis of APS but we acknowledge that missing data on APS is likely and the ICD-10 code for APS has not been validated. To address this, we also performed a sensitivity analysis using medical record-confirmed APS diagnoses in the Stockholm Clinical Cohort data and observed no difference in the relative risk for stroke associated with SLE.

The current study benefits from the inclusion of high quality inpatient and outpatient data as well as stroke information from acute care clinics registered in Riksstroke. Because these registers have nationwide coverage and allowed for follow-up of a relatively large population of individuals diagnosed with SLE for up to 11 years, it was possible to investigate the risk of stroke associated with SLE stratified by patient characteristics. The large sample size also allowed for the examination of stroke subtypes, which have previously been ignored or treated as a composite outcome despite their etiological differences. Our results are further strengthened by testing our findings in a subgroup of clinically confirmed SLE cases demonstrating the robustness of our register-based estimates. We do not have information on race, but previous studies have shown that more than 90\% of SLE patients treated in Swedish university clinics are Caucasian. [24] Our results are generalizable to other Caucasian SLE populations seen in outpatient care in the last decade. In conclusion, younger individuals and women with SLE have an especially higher relative risk than the general population, although their absolute risk remains low. The increased relative risk in the first year after diagnosis highlights a time period where preventative measures could be taken. It is recommended to screen for traditional risk factors at SLE diagnosis and at least annually thereafter,[25] but which of the SLE-specific factors are most important should be assessed in future work. Furthermore, what actions should be taken to modify stroke risk should be clarified so that the burden of stroke can be reduced in this vulnerable population.

\section{Acknowledgments}


Thank you to the Riksstroke Register and to all of those who contributed data to Riksstroke. Thank you to the patients and health care workers who contributed data that was used to make this study possible.

\section{Contributors}

EA and JFS designed the study. EA conducted the analyses and wrote the manuscript. All authors contributed to interpreting the results and revising the manuscript. All authors approved of the final version before submission.

\section{Funding}

This study was funded by STROKE-Riksförbundet (The Swedish National Stroke Foundation). This work was also supported by grants from the County Council of Östergötland, the Swedish Heart-Lung foundation, the Swedish Rheumatism Association, the Swedish Society of Medicine, the King Gustaf V 80 -year foundation and grants provided by the Stockholm County Council (ALF project).

\section{Competing interests}

None declared.

\section{Ethics Approval}

Regional Ethics Review Board in Stockholm for the data linkage (DNR: 2011/920-31/1) and Stockholm clinical cohort (03-556) and Linköping Ethics Review Board for the Linköping clinical cohort (M7508/2008).

\section{REFERENCES}

1. Schoenfeld SR, Kasturi S, Costenbader KH. The epidemiology of atherosclerotic cardiovascular disease among patients with SLE: a systematic review. Seminars in arthritis and rheumatism. 2013 Aug; 43(1):77-95.

2. Symmons DP, Gabriel SE. Epidemiology of CVD in rheumatic disease, with a focus on RA and SLE. Nat Rev Rheumatol. 2011 Jul; 7(7):399-408.

3. Cervera R, Khamashta MA, Font J, Sebastiani GD, Gil A, Lavilla P, et al. Morbidity and mortality in systemic lupus erythematosus during a 10-year period: a comparison of early and late manifestations in a cohort of 1,000 patients. Medicine. 2003 Sep; 82(5):299-308.

4. O'Sullivan M, Bruce IN, Symmons DP. Cardiovascular risk and its modification in patients with connective tissue diseases. Best Pract Res Clin Rheumatol. 2016 Feb; 30(1):81-94.

5. Roubille C, Richer V, Starnino T, McCourt C, McFarlane A, Fleming P, et al. The effects of tumour necrosis factor inhibitors, methotrexate, non-steroidal anti-inflammatory drugs and corticosteroids on cardiovascular events in rheumatoid arthritis, psoriasis and psoriatic arthritis: a systematic review and meta-analysis. Annals of the rheumatic diseases. 2015 Mar; 74(3):480-489. 6. Wannamethee SG, Shaper AG, Perry IJ. Serum creatinine concentration and risk of cardiovascular disease: a possible marker for increased risk of stroke. Stroke; a journal of cerebral circulation. 1997 Mar; 28(3):557-563.

7. Libby P, Ridker PM, Maseri A. Inflammation and atherosclerosis. Circulation. 2002 Mar 5; 105(9):1135-1143.

8. Holmqvist M, Simard JF, Asplund K, Arkema EV. Stroke in systemic lupus erythematosus: a meta-analysis of population-based cohort studies. RMD open. 2015; 1(1):e000168. 9. Mok CC, Ho LY, To CH. Annual incidence and standardized incidence ratio of cerebrovascular accidents in patients with systemic lupus erythematosus. Scandinavian journal of rheumatology. 2009; 38(5):362-368. 
10.

Wang IK, Muo CH, Chang YC, Liang CC, Lin SY, Chang CT, et al. Risks, subtypes, and hospitalization costs of stroke among patients with systemic lupus erythematosus: a retrospective cohort study in Taiwan. The Journal of rheumatology. 2012 Aug; 39(8):1611-1618.

11. Zoller B, Li X, Sundquist J, Sundquist K. Risk of subsequent ischemic and hemorrhagic stroke in patients hospitalized for immune-mediated diseases: a nationwide follow-up study from Sweden. BMC neurology. 2012; 12:41.

12. O'Donnell MJ, Xavier D, Liu L, Zhang H, Chin SL, Rao-Melacini P, et al. Risk factors for ischaemic and intracerebral haemorrhagic stroke in 22 countries (the INTERSTROKE study): a casecontrol study. Lancet. 2010 Jul 10; 376(9735):112-123.

13. Feigin VL, Roth GA, Naghavi M, Parmar P, Krishnamurthi R, Chugh S, et al. Global burden of stroke and risk factors in 188 countries, during 1990-2013: a systematic analysis for the Global Burden of Disease Study 2013. Lancet Neurol. 2016 Aug; 15(9):913-924.

14. Arkema EV, Jonsen A, Ronnblom L, Svenungsson E, Sjowall C, Simard JF. Case definitions in Swedish register data to identify systemic lupus erythematosus. BMJ Open. 2016; 6(1):e007769.

15. Koster M, Asplund K, Johansson A, Stegmayr B. Refinement of Swedish administrative registers to monitor stroke events on the national level. Neuroepidemiology. 2013; 40(4):240-246.

16. Asplund K, Hulter Asberg K, Appelros P, Bjarne D, Eriksson M, Johansson A, et al. The Riks-Stroke story: building a sustainable national register for quality assessment of stroke care. Int J Stroke. 2011 Apr; 6(2):99-108.

17. Frodlund M, Dahlstrom O, Kastbom A, Skogh T, Sjowall C. Associations between antinuclear antibody staining patterns and clinical features of systemic lupus erythematosus: analysis of a regional Swedish register. BMJ Open. 2013 Oct 25; 3(10):e003608.

18. Gustafsson JT, Gunnarsson I, Kallberg H, Pettersson S, Zickert A, Vikerfors A, et al. Cigarette smoking, antiphospholipid antibodies and vascular events in Systemic Lupus Erythematosus. Annals of the rheumatic diseases. 2015 Aug; 74(8):1537-1543.

19. Kohl M, Plischke M, Leffondre K, Heinze G. PSHREG: a SAS macro for proportional and nonproportional subdistribution hazards regression. Computer methods and programs in biomedicine. 2015 Feb; 118(2):218-233.

20. Appelros P, Jonsson F, Asplund K, Eriksson M, Glader EL, Asberg KH, et al. Trends in baseline patient characteristics during the years 1995-2008: observations from Riks-Stroke, the Swedish Stroke Register. Cerebrovascular diseases (Basel, Switzerland). 2010; 30(2):114-119.

21. Grau AJ, Urbanek C, Palm F. Common infections and the risk of stroke. Nat Rev Neurol. 2010 Dec; 6(12):681-694.

22. Esdaile JM, Abrahamowicz M, Grodzicky T, Li Y, Panaritis C, du Berger R, et al. Traditional Framingham risk factors fail to fully account for accelerated atherosclerosis in systemic lupus erythematosus. Arthritis and rheumatism. 2001 Oct; 44(10):2331-2337.

23. Magder LS, Petri M. Incidence of and risk factors for adverse cardiovascular events among patients with systemic lupus erythematosus. Am J Epidemiol. 2012 Oct 15; 176(8):708-719. 24. Jonsen A, Nilsson SC, Ahlqvist E, Svenungsson E, Gunnarsson I, Eriksson KG, et al. Mutations in genes encoding complement inhibitors CD46 and CFH affect the age at nephritis onset in patients with systemic lupus erythematosus. Arthritis Res Ther. 2011; 13(6):R206.

25. Mosca M, Tani C, Aringer M, Bombardieri S, Boumpas D, Brey R, et al. European League Against Rheumatism recommendations for monitoring patients with systemic lupus erythematosus in clinical practice and in observational studies. Annals of the rheumatic diseases. $2010 \mathrm{Jul} ; 69(7): 1269-1274$. 


\section{TABLES}

Table 1. Characteristics of the systemic lupus erythematosus and general population groups at start of follow-up

\begin{tabular}{lrr}
\hline & General population & Systemic Lupus \\
\hline $\mathbf{N}$ & 16,730 & 3,390 \\
\hline Female, \% & 85.0 & 85.1 \\
\hline Age at start of follow-up, mean (SD) & $48.9(17.3)$ & $49.5(17.6)$ \\
\hline Person-years, mean (SD) & $5.1(3.1)$ & $4.8(3.1)$ \\
\hline Country of birth, \% & & \\
\hline$\quad$ Nordic & 89.4 & 85.0 \\
\hline$\quad$ Non-Nordic Europe & 2.6 & 2.5 \\
\hline$\quad$ Non-Europe & 7.9 & 12.5 \\
\hline Education level, \% & & \\
\hline$\quad$ 9 years & 19.4 & 22.8 \\
\hline 10-12 years & 40.0 & 38.4 \\
\hline$>12$ years & 29.7 & 27.0 \\
\hline$\quad$ Missing & 10.9 & 11.9 \\
\hline Comorbidities, \% & & \\
\hline$\quad$ Atrial fibrillation & 2.0 & 3.8 \\
\hline$\quad$ Congestive heart disease & 1.2 & 4.0 \\
\hline Hypertension & 6.4 & 15.7 \\
Diabetes & 2.9 & 4.4 \\
\hline
\end{tabular}

Table 2. Characteristics of individuals with systemic lupus erythematosus and their general population comparators who were diagnosed with incident stroke 2003-2012

\begin{tabular}{lrr}
\hline & General population & Systemic Lupus \\
\hline $\mathbf{N}$ & 304 & 126 \\
\hline Female, \% & 68.1 & 78.6 \\
\hline Age at stroke diagnosis, mean (SD) & $73.3(12.0)$ & $68.4(15.6)$ \\
\hline Minimum, Maximum age at stroke & 32,96 & 20,93 \\
\hline Type of stroke, $\mathbf{n}$ (\%) & & \\
\hline$\quad$ Ischaemic stroke & $228(75.0)$ & $99(78.6)$ \\
\hline$\quad$ Intracerebral haemorrhage & $37(12.2)$ & $11(8.7)$ \\
\hline$\quad$ Subarachnoid haemorrhage & $17(5.6)$ & $5(4.0)$ \\
\hline$\quad$ Unspecified & $22(7.2)$ & $11(8.7)$ \\
\hline $\begin{array}{l}\text { Deceased within 3 months of stroke } \\
\text { diagnosis, } \mathbf{n} \text { (\%) }\end{array}$ & $41(13.5)$ & $24(19.0)$ \\
\hline
\end{tabular}


Table 3. Number of strokes, person-years, incidence rates and hazard ratios for incident stroke (all strokes, ischaemic stroke, intracerebral haemorrhage, subarachnoid haemorrhage and unspecified) comparing SLE to the general population 2003-2013 in Sweden.

\begin{tabular}{|c|c|c|c|c|c|c|c|}
\hline & \multicolumn{2}{|c|}{ General population } & \multicolumn{2}{|r|}{ SLE } & \multirow{2}{*}{$\begin{array}{l}\text { Age- and sex- } \\
\text { adjusted rate } \\
\text { difference } \\
(95 \% \mathrm{Cl})\end{array}$} & \multirow{2}{*}{$\begin{array}{l}\text { Model } 1 \\
\text { Hazard Ratio } \\
(95 \% \mathrm{Cl})\end{array}$} & \multirow{2}{*}{$\begin{array}{l}\text { Model } 2 \\
\text { Hazard Ratio } \\
(95 \% \mathrm{Cl})\end{array}$} \\
\hline & $\begin{array}{l}\text { No. } \\
\text { Strokes }\end{array}$ & $\begin{array}{l}\text { Incidence Rate* } \\
(95 \% \mathrm{Cl})\end{array}$ & $\begin{array}{l}\text { No. } \\
\text { Strokes }\end{array}$ & $\begin{array}{l}\text { Incidence Rate* } \\
(95 \% \mathrm{Cl})\end{array}$ & & & \\
\hline Any Stroke & 304 & $3.5(3.2,4.0)$ & 126 & $7.7(6.5,9.2)$ & $4.4(3.0,5.9)$ & $2.3(1.8,2.8)$ & $2.1(1.7,2.6)$ \\
\hline Ischaemic Stroke & 228 & $2.7(2.3,3.0)$ & 99 & $6.0(5.0,7.4)$ & $3.6(2.3,4.9)$ & $2.4(1.9,3.0)$ & $2.2(1.8,2.8)$ \\
\hline Intracerebral Haemorrhage & 37 & $0.4(0.3,0.6)$ & 11 & $0.7(0.4,1.2)$ & $0.3(-0.2,0.7)$ & $1.6(0.8,3.2)$ & $1.4(0.7,2.9)$ \\
\hline Subarachnoid Haemorrhage & 17 & $0.2(0.1,0.3)$ & 5 & $0.3(0.1,0.7)$ & $0.1(-0.2,0.4)$ & $1.6(0.6,4.3)$ & $1.4(0.5,3.9)$ \\
\hline Unspecified & 22 & $0.3(0.2,0.4)$ & 11 & $0.7(0.4,1.2)$ & $0.5(0.0,0.9)$ & $2.7(1.3,5.5)$ & $2.7(1.3,5.6)$ \\
\hline
\end{tabular}

*Incidence rate per 1000 person-years, confidence interval estimated based on the Poisson distribution.

No. person-years in general population: 85,944 . No. person-years in SLE population: 16,386

Model 1 Cox regression model adjusted for age and sex. Model 2 adjusted for age, sex, education, history of hypertension, diabetes, congestive heart disease and atrial fibrillation. 
Table 4. Number of strokes, person-years, incidence rates, rate differences and hazard ratios for incident ischaemic stroke and intracerebral haemorrhage stratified by sex, age and time since diagnosis 2003-2013 in Sweden.

\begin{tabular}{|c|c|c|c|c|c|c|c|}
\hline & \multicolumn{2}{|c|}{ General population } & \multicolumn{2}{|c|}{ SLE } & \multirow{2}{*}{$\begin{array}{l}\text { Age- and sex- } \\
\text { adjusted rate } \\
\text { difference } \\
(95 \% \mathrm{Cl})\end{array}$} & \multirow{2}{*}{$\begin{array}{c}\text { Model } 1 \\
\text { Hazard Ratio } \\
(95 \% \mathrm{Cl})\end{array}$} & \multirow{2}{*}{$\begin{array}{c}\text { Model } 2 \\
\text { Hazard Ratio } \\
(95 \% \mathrm{Cl})\end{array}$} \\
\hline & $\begin{array}{l}\text { No. Strokes/ } \\
\text { person-years }\end{array}$ & $\begin{array}{c}\text { Incidence Rate* } \\
(95 \% \mathrm{Cl})\end{array}$ & $\begin{array}{l}\text { No. Strokes/ } \\
\text { person-years }\end{array}$ & $\begin{array}{c}\text { Incidence Rate* } \\
(95 \% \mathrm{Cl})\end{array}$ & & & \\
\hline \multicolumn{8}{|l|}{ Ischaemic Stroke } \\
\hline Males & 79 / 12833 & $6.2(4.9,7.7)$ & $22 / 2366$ & $9.3(6.1,14.1)$ & $2.9(-1.1,7.0)$ & $1.5(0.9,2.4)$ & $1.4(0.9,2.2)$ \\
\hline Females & 149 / 73111 & $2.0(1.7,2.4)$ & 77 / 14021 & $5.5(4.4,6.9)$ & $3.7(2.4,5.0)$ & $2.8(2.1,3.7)$ & $2.8(2.1,3.7)$ \\
\hline$<50 y$ & $14 / 46863$ & $0.3(0.2,0.5)$ & 14 / 9189 & $1.5(0.9,2.6)$ & $1.2(0.4,2.1)$ & $5.1(2.5,10.8)$ & $4.6(2.1,9.9)$ \\
\hline $50-<60 y$ & $36 / 16020$ & $2.2(1.6,3.1)$ & $14 / 3016$ & $4.6(2.7,7.8)$ & $2.4(-0.2,4.9)$ & $2.1(1.1,3.9)$ & $1.7(0.9,3.1)$ \\
\hline$\geq 60 \mathrm{y}$ & $178 / 23061$ & $7.7(6.7,8.9)$ & 71 / 4181 & $17.0(13.5,21.4)$ & $9.2(5.1,13.3)$ & $2.2(1.7,2.9)$ & $2.1(1.6,2.8)$ \\
\hline $0-<1 y$ & $27 / 15920$ & $1.7(1.2,2.5)$ & 22 / 3173 & $6.9(4.6,10.5)$ & $1.1(0.5,1.7)$ & $3.9(2.2,6.8)$ & $3.4(1.9,6.1)$ \\
\hline $1-<5 y$ & $125 / 46780$ & $2.7(2.2,3.2)$ & 49 / 8980 & $5.5(4.1,7.2)$ & $1.7(0.8,2.6)$ & $2.1(1.5,2.9)$ & $2.0(1.4,2.8)$ \\
\hline $5-11 y$ & $76 / 23244$ & $3.3(2.6,4.1)$ & $28 / 4232$ & $6.6(4.6,9.6)$ & $1.4(0.4,2.3)$ & $2.3(1.5,3.6)$ & $2.2(1.4,3.4)$ \\
\hline \multicolumn{8}{|l|}{$\begin{array}{l}\text { Intracerebral } \\
\text { Haemorrhage }\end{array}$} \\
\hline Males & 9 / 12833 & $0.7(0.4,1.3)$ & NA & NA & NA & NA & NA \\
\hline Females & $28 / 73111$ & $0.4(0.3,0.6)$ & $10 / 14020$ & $0.7(0.3,1.3)$ & $0.4(-0.1,0.8)$ & $2.0(1.0,4.0)$ & $1.8(0.9,3.7)$ \\
\hline$<60 y$ & $8 / 62883$ & $0.1(0.1,0.3)$ & $5 / 12204$ & $0.41(0.2,1.0)$ & $0.3(-0.1,0.7)$ & $3.3(1.1,10.0)$ & $3.2(1.1,9.9)$ \\
\hline$\geq 60 y$ & $29 / 23061$ & $1.3(0.9,1.8)$ & $6 / 4181$ & $1.4(0.6,3.2)$ & $0.17(-1.1,1.4)$ & $1.2(0.5,2.8)$ & $1.0(0.4,2.4)$ \\
\hline$<5 y$ & $24 / 62700$ & $0.4(0.3,0.6)$ & $6 / 12154$ & $0.5(0.2,1.1)$ & $0.10(-0.2,0.4)$ & $1.3(0.5,3.2)$ & $1.1(0.5,2.8)$ \\
\hline $5-11$ y & $13 / 23244$ & $0.6(0.3,1.0)$ & $5 / 4232$ & $1.2(0.5,2.8)$ & $0.2(-0.2,0.6)$ & $2.3(0.8,6.3)$ & $2.2(0.8,6.2)$ \\
\hline
\end{tabular}

NA marks cell counts less than 5 , corresponding incidence rates and hazard ratios were therefore not estimated.

Incidence rates per 1000 person-years. Confidence intervals calculated using the Poisson distribution.

Model 1 Cox regression model adjusted for age and sex. Model 2 adjusted for age, sex, education, history of hypertension, diabetes, congestive heart disease diagnoses and atrial fibrillation. 
